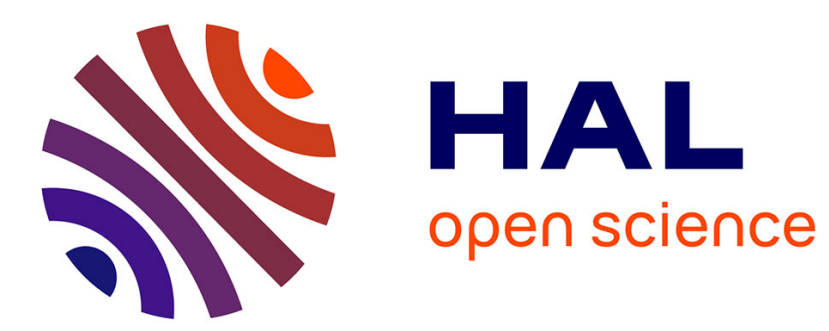

\title{
Des femmes en capacité d'agir. Introduction au dossier \\ Marie Augier
}

\section{To cite this version:}

Marie Augier. Des femmes en capacité d'agir. Introduction au dossier. Archimède: archéologie et histoire ancienne, 2018, Archimède $\mathrm{n}^{\circ} 5$. Archéologie et histoire ancienne, 5, pp.108-112. halshs01825266

\section{HAL Id: halshs-01825266 https://shs.hal.science/halshs-01825266}

Submitted on 29 Jun 2018

HAL is a multi-disciplinary open access archive for the deposit and dissemination of scientific research documents, whether they are published or not. The documents may come from teaching and research institutions in France or abroad, or from public or private research centers.
L'archive ouverte pluridisciplinaire HAL, est destinée au dépôt et à la diffusion de documents scientifiques de niveau recherche, publiés ou non, émanant des établissements d'enseignement et de recherche français ou étrangers, des laboratoires publics ou privés. 


\section{ARCHIMĖDE N'5

1 DOSSIER THÉMATIQUE : HUMOEROTICA

\section{ACTUALITÉ DE LA RECHERCHE : DES FEMMES PUBLIQUES. GENRE, VISIBILITÉ ET SOCIABILITÉ DANS L’ANTIQUITÉ GRECQUE ET ROMAINE}

108 Marie AUGIER

Des femmes en capacité d'agir. Introduction au dossier

113 Louise BRUIT ZAIDMAN

«L'enfant du foyer ». Des statues pour les filles et les garçons initiés à Éleusis au nom de la cité

124 Hélène CASTELLI

Pèlerines à Épidaure. Femmes, guérison et publicité dans un sanctuaire panhellénique au IV siècle av. J.-C.

134 Marie-Laure SRONEK

Des femmes invisibles dans l'Athènes classique ? Les effets du travail pour une redéfinition de la place des femmes dans la vie publique

145 Annalisa PARADISO

Femmes lydiennes et crises dynastiques

\section{Sandra PÉRÉ-NOGUÈS}

Sur les traces de Philistis, « reine » de Syracuse : quelques réflexions sur la visibilité des femmes dans les sources monétaires

165 Christine HUE-ARCÉ

Grec(que)s contre Égyptien(ne)s dans les enteuxeis ptolémaïques : la question du genre dans les $P$. Enteux. 79 et $P$. Enteux. 82

175 Claudia BELTRÃO and Patricia HORVAT

The Name of the Vestal, or When a Vestal is Named

185 VARIA

246 LA CHRONIQUE D'ARCHIMÈDE 


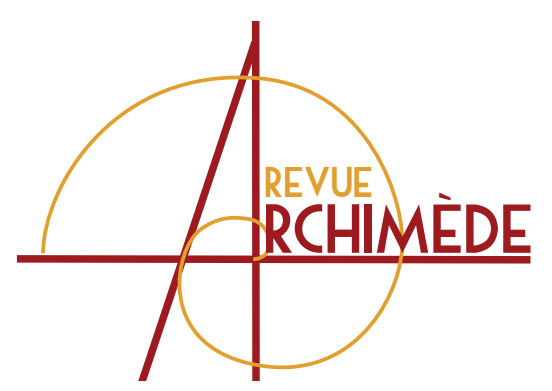

\title{
ACTUALITÉ DE LA RECHERCHE DES FEMMES PUBLIQUES. GENRE, VISIBILITÉ ET SOCIABILITÉ DANS L'ANTIQUITÉ GRECQUE ET ROMAINE
}

\author{
DES FEMMES EN CAPACITÉ D’AGIR \\ INTRODUCTION AU DOSSIER
}

\author{
Marie AUGIER \\ Docteure en histoire grecque \\ Chercheuse associée UMR 7044 \\ marie.augier@gmail.com
}

\section{RÉSUMÉ}

Le dossier regroupe des articles mettant en lumière la place des femmes dans l'Antiquité gréco-romaine. Dans les sources convoquées par les auteurs - documents épigraphiques, papyrus, monnaies et textes issus de la tradition manuscrite -, des femmes sont nommées parce qu'elles accomplissaient des actions qui les rendaient visibles au sein de leur communauté civique. Cette enquête montre que, loin d'être passives ou recluses, les femmes investissaient l'espace public et

MotS-CLÉS

Histoire ancienne, femmes,

genre,

Grèce,

Rome. participaient activement à la vie de la cité : leurs actions étaient jugées dignes d'être affichées et conservées dans la mémoire collective.
These papers shed light on the place held by the women of Greco-Roman Antiquity. The authors draw on various sources (epigraphic documents, papyrus, coins, literature) where women are named because their actions have marked them out among the civic community. The study reveals how, far from being passive or secluded, women took active part in socialization and engaged in the public space, where their actions were thought to be worth displaying and preserving in the collective memory.
KeYWORDS

Ancient history, women, gender, Greece, Rome. 
Qu'entend-on par « femmes publiques »? L'expression, lorsqu'elle se décline au masculin, désigne sans ambiguïté des hommes dont les actions sont visibles dans la communauté à laquelle ils appartiennent. Pourtant, cette même expression au féminin prend un sens différent : elle peut prêter à confusion et suggérer, selon un usage vieilli, qu'il s'agit de prostituées. Dans le domaine des travaux sur l'Antiquité gréco-romaine, la tradition historiographique tend généralement, lorsqu'il s'agit de femmes, à associer visibilité et mauvaise réputation : les femmes citées dans certaines sources [1] seraient, selon cette lecture, considérées par les Anciens eux-mêmes comme peu « recommandables ». Le corollaire de ce constat est alors que les femmes « recommandables » seraient, quant à elles, cantonnées dans l'espace domestique. Dans son Économique, Xénophon évoque en effet cette répartition des espaces lorsqu'il présente l'oikos d'Ischomaque : « La coutume déclare convenables les occupations pour lesquelles la divinité a donné à chacun le plus de capacités naturelles. Pour la femme, il est plus convenable de rester à la maison que de passer son temps dehors, et il l'est moins pour l'homme de rester à la maison que de s'occuper des travaux de l'extérieur [2]. » L'épouse d'Ischomaque n'est jamais nommée dans l'ouvrage, et cette absence de nomination a été interprétée

[1] Il s'agit en particulier des discours des orateurs (voir analyse infra).

[2] Xénophon, Économique, VII, 30 (trad. P. Chantraine). Xénophon justifie la prédisposition de la gunê aux travaux de la maison et celle de l'anêr aux travaux de l'extérieur par leur nature respective, telle que l'ont définie les dieux ; à ce sujet, VII, 19-23. Cf. également Aristote, Économique, livre I, 3, [1344a].

[3] Sur la question de la visibilité du nom des femmes, Boehringer, Grand-Clément, PÉré-Noguès \& Sebillotte-Cuchet, 2015.

[4] VernanT (1963) 1990, p. 162. Il ajoute : « La femme est donc dans son domaine à la maison. C'est là qu'est sa place ; en principe elle n'en doit pas sortir. comme une preuve de l'invisibilité des femmes, entendues ici comme les épouses de citoyens [3]. Dans les années 1960, Jean-Pierre Vernant interprétait l'espace grec selon une distinction nette entre l'espace des hommes et celui des femmes, en s'appuyant sur la complémentarité entre deux figures divines : Hestia et Hermès. Il opposait ainsi « l'espace domestique, espace fermé, pourvu d'un toit (protégé) [...] à connotation féminine » et « l'espace du dehors, du grand air, à connotation masculine [4]. » À la fin des années 70, David Schaps constate ainsi que les orateurs taisaient le nom des femmes bien nées dans leurs plaidoyers judiciaires, afin de ne pas salir leur réputation [5]. Les travaux de Nicole Loraux [6], s'appuyant surtout sur I'analyse des discours portant sur la Grèce classique et archaïque (épopées, tragédies, traités philosophiques ou techniques), reprennent et précisent cette dichotomie.

Pourtant, d'autres travaux en histoire des femmes et du genre ont montré que les femmes qui paraissaient « en public » [7] et dont les noms étaient cités n'étaient pas toutes des «femmes publiques » au sens dépréciatif du terme : les donatrices [8] et les prêtresses [9] honorées par les cités n'en sont qu'un infime exemple. Ces travaux s'appuient notamment sur des documents de nature archéologique (épigraphie et papyrus), datant de périodes plus récentes :

L'homme représente au contraire dans l'oikos, l'élément centrifuge : c'est à lui de quitter l'enclos rassurant du foyer pour affronter les fatigues, les dangers, les imprévus de l'extérieur, à lui d'établir le contact avec le dehors, d'entrer en commerce avec l'étranger. »

[5] SCHAPS 1977.

[6] Par exemple LoRAux 1989.

[7] C'est le titre choisi par Anne Bielman dans son recueil de documents en 2002 (BiELman 2002) : Femmes en public dans le monde hellénistique.

[8] Sur Archippè de Kyme : SAVALLI-LeSTRADE 2003.

[9] Sur les prêtresses : KRON 1993 ; GEORGOUDI 2003 et 2005 ; CONNELLY 2007 ; Augier 2017. 
ils compensent l'athénocentrisme des sources issues de la tradition manuscrite et s'intéressent à des domaines d'activité variés. L'analyse d'une telle documentation s'inscrit dans la lignée de l'entreprise éditoriale de l'Histoire des femmes en Occident [10], qui avait pour but de dénoncer Les Silences de I'histoire [11], une histoire qui semblait se réaliser uniquement par l'action des hommes [12].

Depuis les années 2000, I'outil du genre offre de nouvelles perspectives aux historien-ne-s de I'Antiquité. Il permet d'étudier la manière dont les sociétés interprètent la différence des sexes en mettant au jour la construction sociale qui lui est associée. Les travaux en France, aux États-Unis et désormais en Amérique latine se multiplient [13]. L'ouvrage, dirigé par Sandra Boehringer et Violaine Sebillotte et publié en 2011, Hommes et femmes dans I'Antiquité grecque et romaine. Le genre : méthode et documents, propose une synthèse de ces nouveaux acquis par des études de documents variés et suggère, après d'autres [14], la richesse des anaIyses possibles. Ce travail collectif a servi de point de départ méthodologique à un vaste programme de recherche, lancé en 2013 : le projet « Eurykleia. Celles qui avaient un nom »[15], porté par quatre laboratoires, les laboratoires ANHIMA (UMR 8210), ARChimede (UMR 7044), TRACES (UMR 5608) et PLH (EA 4601). L'objectif de ce projet scientifique consiste à réunir les données sur les noms des femmes pour mettre au jour les diverses appréciations de la différence des sexes dans les sociétés antiques, en faisant jouer le paramètre de la diversité des contextes d'apparition de leur nom. Le but du projet est d'élaborer une base de données qui fournira à la communauté scientifique un outil statistique nécessaire à une étude des femmes et du genre, en intégrant le critère des pratiques sociales dans lesquelles le nom apparaît. Depuis le lancement du projet, plusieurs volumes et travaux collectifs ont vu le jour [16]. La collaboration avec I'équipe Nereida rattachée aux universités brésiliennes de Rio de Janeiro (UNIRIo et Université fédérale Fluminense) trouve ici sa première expression dans une revue française.

Ainsi, ce dossier « Femmes publiques » est un nouveau jalon dans ce programme collectif ambitieux. Les articles rassemblés proposent d'interroger une documentation large, mentionnant le nom de femmes grecques et romaines : textes épigraphiques, monnaies et textes issus de la tradition manuscrite. Par la mention de leur nom associé à une fonction ou à des actions spécifiques, ces femmes acquièrent une visibilité et deviennent des femmes publiques, c'est-à-dire des femmes dont les actions dans la communauté à laquelle elles appartiennent sont accomplies au grand jour et jugées dignes d'être retranscrites, affichées et gravées dans la mémoire collective. Par leur présence dans l'espace public, - qu'il s'agisse de l'agora, des sanctuaires, des tribunaux ou de la cour royale ou impériale -, ces femmes nommées ne font pas seulement partie d'une communauté, elles en sont aussi les actrices et participent à la sociabilité du groupe civique. Chacun des articles qui composent le dossier évoque des parcours de femmes grecques et romaines dans le champ du sacré, du politique, du commerce ou de la mobilité. L'analyse de la documentation écrite cherche à mettre en lumière ce que le contexte d'apparition du nom de ces femmes nous apprend sur leur implication dans la vie des cités et royaumes du monde grec et romain. À quelle catégorie d'âge appartiennent-elles ? Quel est leur statut social ? Quelle est leur fonction ? Quelles actions émanant de ces femmes sont évoquées ? Leurs actions sont-elles valorisées ou non ? Les noms des femmes grecques ou romaines qui ont été conservés servent de point de départ pour observer la façon dont le genre interagit avec d'autres critères comme le statut politique, socio-économique ou encore les liens de parenté. Une telle démarche permet ainsi, entre autre, de réinterroger la catégorisation de «division de sexes » en la confrontant aux catégories contemporaines si complexes et mouvantes de privé et de public [17].

[10] DUBY \& PERROT (1990-1992) 2002.

[11] PERROT 1998.

[12] Voir aussi SchmitT-PANTEL 1994-1995 et dans la continuité de ces travaux 2009.

[13] Les articles de Pauline Schmitt-Pantel (réunis dans SchmitT-PANTEL 2009) offrent une mise en perspective, depuis la France, de l'histoire des femmes et du genre dans les sciences de l'Antiquité depuis les années 1980. La bibliographie réunie dans les différentes contributions du Companion to Women in the Ancient Word (JAMES \& DiLLON 2012) permet un large balayage thématique, prioritairement du point de vue de I'historiographie anglophone. Voir également la bibliographie et les travaux rassemblés dans la revue Iphis (FEICHTINGER \& WöHRLE 2002-2010; FEICHTINGER, FUHRER, \& WÖHRLE 2014-2018).

[14] Sur ces questions, SCOTT 2012 ; ERNOULT \& Sebillotte 2007 ; Boehringer \& Sebillotte 2011 et 2013.

[15] « Eurykleia, celles qui avaient un nom » : http:// eurykleia.hypotheses.org/

[16] Boehringer \& Sebillotte 2013 ; Boehringer, Grand-Clément, Péré-Noguès \& Sebillotte 2015.

[17] Sur la remise en cause de l'opposition « public/ privé », voir SCHMITT-PANTEL 2009, p. 109-112. 
La première étude, celle de Louise Bruit, met au jour la valeur sociale et la place importante des femmes dans la transmission des charges religieuses publiquement revendiquée ; elle évoque les paides aph'hestias, ces enfants, filles et garçons, élus parmi les grandes familles athéniennes pour remplir une fonction prestigieuse à Éleusis. Ces enfants, quel que soit leur sexe, en devenant « enfants du foyer », remplissent un des nombreux services religieux qu'ils pouvaient rendre à la cité. La publicité faite à leur nom sur des bases de statues les rend ainsi visibles tout en participant à la glorification familiale. Marie-Laure Sronek quant à elle s'intéresse à une tout autre catégorie de femmes athéniennes, moins bien nées : les travailleuses de l'Athènes classique, dont les noms apparaissent sur différents supports : stèles funéraires, dédicaces, tablettes de malédiction. Ces travailleuses, qui exercent des métiers fort divers, participent ainsi à leur échelle aux échanges de leur cité, tout en investissant I'espace public. Elles sont donc loin de l'image traditionnellement dévolue aux Athéniennes, censées rester à l'intérieur de l'oikos. La contribution d'Hélène Castelli concerne les Iamata d'Épidaure, inscriptions qui exposent le nom de suppliantes d'Asclépios n'hésitant pas à entreprendre le voyage pour consulter le dieu - un indice de leur statut social privilégié. L'auteure étudie les critères de nomination des pèlerines, majoritairement des femmes adultes, ainsi que la raison de leur venue. Analisa Paradiso se penche dans son article sur les sources issues de la tradition manuscrite, plus particulièrement Xanthos de Lydie, et s'attache à montrer en quoi des femmes lydiennes de sang royal jouent un rôle lors des renversements dynastiques des Atyades, des Héraclides et des Mermnades. Elle met ainsi au jour un schéma topique faisant jouer leur sexualité dans ces crises dynastiques. Sandra Péré-Noguès s'appuie quant à elle sur des sources à la fois épigraphiques et monétaires pour montrer le rôle politique de Philistis, l'épouse de Hiéron II de Syracuse. Celle-ci participe en effet à la renommée de la famille royale par la publicité qui lui est faite au théâtre ou par l'émission de monnaies à son effigie. Par son statut de basilissa ainsi exposé, elle joue un rôle public au sein de sa cité. C'est en Égypte ptolémaïque que nous conduit pour sa part Christine Hue-Arcé. Elle étudie deux pétitions grecques du Fayoum datées du III $^{\mathrm{e}} \mathrm{s}$. av. J.-C., des plaintes adressées au souverain d'un Grec contre une Égyptienne et d'une Grecque contre un Égyptien. Elle s'interroge sur les logiques liées au genre et/ou à l'origine géographique et sociale des personnes concernées par la plainte. Enfin, Claudia Beltrão et Patricia Horvat s'intéressent aux Vestales nommées dans les écrits de Tite-Live pour montrer comment la publicité faite à certaines femmes peut être associée à des actions répréhensibles. En effet, les Vestales nommées sont celles qui sont accusées criminellement d'incestum.

Les différents articles présentent des femmes qui sont en capacité d'agir : qu'elles exercent un service religieux ou leur rôle de reine, qu'elles voyagent, travaillent, complotent, commettent un acte délictueux ou portent plainte, les actions de ces femmes sont rendues publiques et affichées. Elles les rendent visibles au sein de leur communauté, et pour la postérité. Parce que ces femmes agissent pour des motivations personnelles, professionnelles ou familiales, en tant que détentrices d'une charge publique ou non, leurs noms ont été jugés dignes, au même titre que ceux des hommes, d'être affichés et conservés dans la mémoire collective. 
Augrer, Marie, 2017, « Nommer les femmes en Grèce ancienne : le cas des prêtresses athéniennes », Clio. Femmes, Genre, Histoire, tome 45, p. 33-59.

Bielman, Anne, 2002, Femmes en public dans le monde hellénistique : IVe- $\mathrm{I}^{\mathrm{er}} \mathrm{s}$. av. J.-C., Paris.

Boehringer, Sandra \& Sebillotte-Cuchet, Violaine (dir.), 2011, Hommes et femmes dans l'Antiquité grecque et romaine. Le genre : méthode et documents, Paris.

Boehringer, Sandra \& Sebillotte-Cuchet, Violaine (dir.), 2013, Des femmes en action. L'individu et la fonction en Grèce antique, Mètis, Anthropologie des mondes grecs anciens Hors Série 1, Paris - Athènes.

Boehringer, Sandra, Grand-Clément, Adeline, Péré-Noguès, Sandra \& Sebillotte-Cuchet, Violaine (éd.), 2015, Laisser son nom : femmes et actes de mémoire dans les sociétés anciennes, Pallas 99, p. 9-131.

Boehringer, Sandra, Ernoult, Nathalie \& Sebillotte-Cuchet, Violaine (éd.), 2018, Sortir du gynécée. Un nouveau regard sur la Grèce antique, exposition virtuelle éditée par MUSEA, musée d'histoire des femmes et du genre : http://musea.univ-angers.fr/exhibits/show/sortir-du-gynecee/presentation.

Connelly, Joan Breton, 2007, Portrait of a Priestess, Women and Ritual in Ancient Greece, Princeton - Oxford.

Duby, Georges \& Perrot, Michelle (dir.), 2002, Histoire des femmes en Occident, 5 vol. (1 ${ }^{\text {re }}$ éd. 1990-1992), Paris. Ernoult, Nathalie \& Sebillotte-Cuchet, Violaine (éd.), 2007, Problèmes du genre en Grèce ancienne, Paris.

FeIChTINGeR, Barbara, WöhRLE, Georg (éd.), 2002-2010, Gender studies in den Altertumswissenschaften, IPHIS, Beiträge zur altertumswissenschaftlichen Genderforschung, Trier, Band 1-5.

Feichtinger, Barbara, Fuhrer, Therese \& Wöhrle, Georg (éd.), 2014-2018, Gender studies in den Altertumswissenschaften, IPHIS, Beiträge zur altertumswissenschaftlichen Genderforschung, Trier, Band 6-8.

GeORGOUdI, Stella, 2003, « Lysimachè, la prêtresse », dans Nicole Loraux (dir.), La Grèce au féminin, Paris, p. 167-216. GeORGoudr, Stella, 2005, «Athanathous therapeuein. Réflexions sur des femmes au service des dieux», dans Véronique Dasen \& Marcel Piérart (éd.), Kernos, suppl. 15, Les cadres «privés » et « publics » dans la religion grecque antique, Centre International d'Étude de la Religion Grecque antique, Liège, p. 69-82.

JAmes, Sharon L. \& Dillon Sheila, 2012, A Companion to Women in the Ancient Word, Malden.

KRON, Uta, 1993, «Priesthoods, Dedications and Evergetism; What Part Did Religion Play in the Political and Social Status of Greek Women? », dans Pontus Hellström \& Brita Alroth (éd.), Religion and Power in the Ancient Greek World, Proceedings of the Uppsala Symposium, p. 139-182.

LoRAuX, Nicole, 1989, Les expériences de Tirésias. Le féminin et l'homme grec, Paris.

Perrot, Michelle, 1998, Les femmes ou les silences de l'histoire, Paris.

Savalli-Lestrade, Ivana, 2003, « Archippè de Kymè, la bienfaitrice », dans Nicole Loraux (dir.), La Grèce au féminin, Paris (Histoire 57), p. 249-295.

Schaps David, 1977, «The Woman Least Mentioned: Etiquette And Women's Names », Classical Quarterly 27. 2, p. $323-330$. Schmitt Pantel, Pauline, 1994-1995, «Autour d'une anthropologie des sexes. À propos de la femme sans nom d'Ischomaque », Mètis 9-10, p. 299-305

Schmitt Pantel, Pauline, 2009, Aithra et Pandora, Femmes, Genre et Cité dans la Grèce antique, Paris.

ScotT, Joan W., 2012, De l'utilité du genre, Paris.

Sebillotte-Cuchet, Violaine et alii, 2016, Carnet de recherche Eurykleia. Celles qui avaient un nom :

https://eurykleia.hypotheses.org/.

Vernant, Jean-Pierre, 1990, « Hestia-Hermès. Sur l'expression religieuse de l'espace et du mouvement chez les Grecs », Mythe et Pensée chez les Grecs, études de psychologie historique, Paris (1 $1^{\text {re }}$ éd. L'Homme 3.3, 1963, p. 12-50). 